\title{
Homoclinic orbits for asymptotically linear discrete Hamiltonian systems
}

\section{Xiaoping Wang*}

\section{*Correspondence:} wxp31415@sina.com Department of Mathematics, Xiangnan College, Chenzhou, Hunan 423000, P.R. China

\begin{abstract}
We study the existence of homoclinic solutions for the following second-order self-adjoint discrete Hamiltonian system: $\Delta[p(n) \Delta u(n-1)]-L(n) u(n)+\nabla W(n, u(n))=0$, where $p(n), L(n)$, and $W(n, x)$ are $N$-periodic in $n$, and $\nabla W(n, x)$ is asymptotically linear in $x$ as $|x| \rightarrow \infty$.

MSC: 39A11;58E05; 70H05
\end{abstract}

Keywords: homoclinic solution; discrete Hamiltonian system; asymptotically linear; strongly indefinite functional

\section{Introduction}

Discrete Hamiltonian systems can be applied in many areas, such as physics, chemistry, and so on. For more discussions on discrete Hamiltonian systems, we refer the reader to $[1,2]$. In this paper, we consider the second-order self-adjoint discrete Hamiltonian system

$$
\triangle[p(n) \triangle u(n-1)]-L(n) u(n)+\nabla W(n, u(n))=0,
$$

where $n \in \mathbb{Z}, u \in \mathbb{R}^{\mathcal{N}}, \Delta u(n)=u(n+1)-u(n)$ is the forward difference, $p, L: \mathbb{Z} \rightarrow \mathbb{R}^{\mathcal{N} \times \mathcal{N}}$ and $W: \mathbb{Z} \times \mathbb{R}^{\mathcal{N}} \rightarrow \mathbb{R}$.

As usual, we say that a solution $u(n)$ of system (1.1) is homoclinic (to 0$)$ if $u(n) \rightarrow 0$ as $n \rightarrow \pm \infty$. In addition, if $u(n) \not \equiv 0$ then $u(n)$ is called a nontrivial homoclinic solution.

In recent years, several authors studied homoclinic orbits for system (1.1) or its special forms via critical point theory. For example, see [3-18]. We emphasize that in all these papers the nonlinear term was assumed to be superlinear or sublinear at infinity. To the best of our knowledge, the existence of homoclinics for asymptotically linear discrete Hamiltonian systems has not been previously studied.

In this paper, we assume that $p(n)$ and $L(n)$ are $N$-periodic $\mathcal{N} \times \mathcal{N}$ real symmetric matrices. Let $\mathcal{A}$ is an operator defined as follows:

$$
(\mathcal{A} u)(n)=\triangle[p(n) \triangle u(n-1)]-L(n) u(n), \quad \forall n \in \mathbb{Z} .
$$

Then it is easy to check that $\mathcal{A}$ is a bounded self-adjoint operator in $l^{2}\left(\mathbb{Z}, \mathbb{R}^{\mathcal{N}}\right)$, where $l^{2}\left(\mathbb{Z}, \mathbb{R}^{\mathcal{N}}\right)$ is defined in Section 2. By the Floquet theorem, it is easy to verify that $\mathcal{A}$ has only continuous spectrum $\sigma(\mathcal{A})$, which is a union of bounded closed intervals.

(C) 2015 Wang; licensee Springer. This is an Open Access article distributed under the terms of the Creative Commons Attribution License (http://creativecommons.org/licenses/by/4.0), which permits unrestricted use, distribution, and reproduction in any medium, provided the original work is properly credited. 
When $p(n)$ and $L(n)$ are positive definite, $\sigma(\mathcal{A}) \subset(0,+\infty)$. In this case, the mountain pass theorem of Ambrosetti and Rabinowitz is a very useful tool for finding critical points of the energy functionals associated to (1.1). However, when $p(n)$ or $L(n)$ is not positive definite, 0 is a saddle point rather than a local minimum of the functional associated to (1.1), which is strongly indefinite. This case is difficult because the mountain-pass reduction of the definite case is not available, and it is not known if the Palais-Smale sequences are bounded. We choose this case as the object of the present paper.

To state our results, we first introduce the following assumptions:

(PL) $p(n)$ and $L(n)$ are $N$-periodic $\mathcal{N} \times \mathcal{N}$ real symmetric matrices, and

$$
\sup [\sigma(\mathcal{A}) \cap(-\infty, 0)]:=\underline{\Lambda}<0<\bar{\Lambda}:=\inf [\sigma(\mathcal{A}) \cap(0, \infty)] ;
$$

(W1) $W(n, x)$ is continuously differentiable in $x$ for every $n \in \mathbb{Z}, W(n, 0)=0$,

$W(n, x) \geq 0$, and $W(n, x)$ is $N$-periodic in $n$;

(W2) $\nabla W(n, x)=o(|x|)$ as $|x| \rightarrow 0$ uniformly for $n \in \mathbb{Z}$;

(W3) $W(n, x)=\frac{1}{2} M(n) x \cdot x+W_{\infty}(n, x)$, where $M(n)$ is an $N$-periodic $\mathcal{N} \times \mathcal{N}$ real symmetric matrix, $\inf _{n \in \mathbb{Z},|x|=1} M(n) x \cdot x>\bar{\Lambda}, \nabla W_{\infty}(n, x)=o(|x|)$ as $|x| \rightarrow \infty$, uniformly for $n \in \mathbb{Z}$;

(W4) $\widetilde{W}(n, x):=\frac{1}{2} \nabla W(n, x) \cdot x-W(n, x) \geq 0, \forall(n, x) \in \mathbb{Z} \times \mathbb{R}^{\mathcal{N}}$, and there exists a $\delta_{0} \in\left(0, \Lambda_{0}\right)$ with $\Lambda_{0}=\min \{-\underline{\Lambda}, \bar{\Lambda}\}$ such that

$$
\frac{|\nabla W(n, x)|}{|x|} \geq \Lambda_{0}-\delta_{0} \Rightarrow \widetilde{W}(n, x) \geq \delta_{0} .
$$

Now, we are ready to state the main result of this paper.

Theorem 1.1 Assume that $p, L$, and $W$ satisfy (PL), (W1), (W2), (W3), and (W4). Then system (1.1) possesses a nontrivial homoclinic solution.

Remark 1.2 The following functions satisfy (W1)-(W4):

$$
\begin{aligned}
& W(n, x)=a(n)|x|^{2}\left[1-\frac{1}{\ln (e+|x|)}\right], \\
& W(n, x)=\int_{0}^{|x|} \alpha(n, s) s \mathrm{~d} s
\end{aligned}
$$

where $a(n)$ and $\alpha(n, s)$ are $N$-periodic positive function in $n, \alpha(n, s)$ is non-decreasing for $s \in[0, \infty), \alpha(n, s) \rightarrow 0$ as $s \rightarrow 0$ and $\alpha(n, s) \rightarrow b(n)$ as $s \rightarrow \infty$ with $\inf _{\mathbb{Z}} b>\bar{\Lambda}$, uniformly in $n \in \mathbb{Z}$.

\section{Proof of theorem}

Let

$$
S=\left\{\{u(n)\}_{n \in \mathbb{Z}}: u(n) \in \mathbb{R}^{\mathcal{N}}, n \in \mathbb{Z}\right\} .
$$

As usual, for $1 \leq q<\infty$, set

$$
l^{q}\left(\mathbb{Z}, \mathbb{R}^{\mathcal{N}}\right)=\left\{u \in S: \sum_{n \in \mathbb{Z}}|u(n)|^{q}<\infty\right\}
$$


and

$$
l^{\infty}\left(\mathbb{Z}, \mathbb{R}^{\mathcal{N}}\right)=\left\{u \in S: \sup _{n \in \mathbb{Z}}|u(n)|<\infty\right\}
$$

and their norms are defined by

$$
\begin{aligned}
& \|u\|_{q}=\left(\sum_{n \in \mathbb{Z}}|u(n)|^{q}\right)^{1 / q}, \quad \forall u \in l^{q}\left(\mathbb{Z}, \mathbb{R}^{\mathcal{N}}\right) ; \\
& \|u\|_{\infty}=\sup _{n \in \mathbb{Z}}|u(n)|, \quad \forall u \in l^{\infty}\left(\mathbb{Z}, \mathbb{R}^{\mathcal{N}}\right),
\end{aligned}
$$

respectively. In particular, $l^{2}\left(\mathbb{Z}, \mathbb{R}^{\mathcal{N}}\right)$ is a Hilbert space with the following inner product:

$$
(u, v)_{l^{2}}=\sum_{n \in \mathbb{Z}} u(n) \cdot v(n), \quad \forall u, v \in l^{2}\left(\mathbb{Z}, \mathbb{R}^{\mathcal{N}}\right) .
$$

Let $\left\{\mathcal{E}(\lambda):-a_{0} \leq \lambda \leq b_{0}\right\}$ and $|\mathcal{A}|$ be the spectral family and the absolute value of $\mathcal{A}$, respectively, and $|\mathcal{A}|^{1 / 2}$ be the square root of $|\mathcal{A}|$. Set $\mathcal{U}=$ id $-\mathcal{E}(0)-\mathcal{E}(0-)$. Then $\mathcal{U}$ commutes with $\mathcal{A},|\mathcal{A}|$ and $|\mathcal{A}|^{1 / 2}$, and $\mathcal{A}=\mathcal{U}|\mathcal{A}|$ is the polar decomposition of $\mathcal{A}$ (see [19, Theorem 4.3.3]).

As in [20], let $E=l^{2}\left(\mathbb{Z}, \mathbb{R}^{\mathcal{N}}\right)$ and

$$
E^{-}=\mathcal{E}(0) E, \quad E^{+}=[\mathrm{id}-\mathcal{E}(0)] E .
$$

For any $u \in E$, it is easy to see that

$$
u^{-}:=\mathcal{E}(0) u \in E^{-}, \quad u^{+}:=[\mathrm{id}-\mathcal{E}(0)] u \in E^{+}, \quad u=u^{-}+u^{+},
$$

and

$$
\mathcal{A} u^{-}=-|\mathcal{A}| u^{-}, \quad \mathcal{A} u^{+}=|\mathcal{A}| u^{+}, \quad \forall u \in E .
$$

Let

$$
(u, v)=\left(|\mathcal{A}|^{1 / 2} u,|\mathcal{A}|^{1 / 2} v\right)_{l^{2}}, \quad \forall u, v \in E .
$$

Then $E$ is a Hilbert space with the above inner product, and the corresponding norm is

$$
\|u\|=\left\||\mathcal{A}|^{1 / 2} u\right\|_{2}, \quad \forall u \in E .
$$

By virtue of (2.1)-(2.4), one has the decomposition $E=E^{-} \oplus E^{+}$orthogonal with respect to both $(\cdot, \cdot)_{l^{2}}$ and $(\cdot, \cdot)$. Moreover,

$$
-\underline{\Lambda}\left\|u^{-}\right\|_{2}^{2} \leq\left\|u^{-}\right\|^{2} \leq a_{0}\left\|u^{-}\right\|_{2}^{2}, \quad \bar{\Lambda}\left\|u^{+}\right\|_{2}^{2} \leq\left\|u^{+}\right\|^{2} \leq b_{0}\left\|u^{+}\right\|_{2}^{2}, \quad \forall u \in E,
$$

and

$$
\Lambda_{0}\|u\|_{2}^{2} \leq\|u\|^{2} \leq \max \left\{a_{0}, b_{0}\right\}\|u\|_{2}^{2}, \quad \forall u \in E
$$


Let $X$ be a real Hilbert space with $X=X^{-} \oplus X^{+}$and $X^{-} \perp X^{+}$. For a functional $\varphi \in$ $C^{1}(X, \mathbb{R}), \varphi$ is said to be weakly sequentially lower semi-continuous if for any $u_{k} \rightarrow u$ in $X$ one has $\varphi(u) \leq \liminf _{n \rightarrow \infty} \varphi\left(u_{k}\right)$, and $\varphi^{\prime}$ is said to be weakly sequentially continuous if $\lim _{k \rightarrow \infty}\left\langle\varphi^{\prime}\left(u_{k}\right), v\right\rangle=\left\langle\varphi^{\prime}(u), v\right\rangle$ for each $v \in X$.

Lemma 2.1 ([21, Theorem 2.1]) Let $X$ be a real Hilbert space with $X=X^{-} \oplus X^{+}$and $X^{-} \perp X^{+}$, and let $\varphi \in C^{1}(X, \mathbb{R})$ of the form

$$
\varphi(u)=\frac{1}{2}\left(\left\|u^{+}\right\|^{2}-\left\|u^{-}\right\|^{2}\right)-\psi(u), \quad u=u^{-}+u^{+} \in X^{-} \oplus X^{+} .
$$

Suppose that the following assumptions are satisfied:

(LS1) $\psi \in C^{1}(X, \mathbb{R})$ is bounded from below and weakly sequentially lower semi-continuous;

(LS2) $\psi^{\prime}$ is weakly sequentially continuous;

(LS3) there exist $r>\rho>0$ and $e \in X^{+}$with $\|e\|=1$ such that

$$
\kappa:=\inf \varphi\left(S_{\rho}^{+}\right)>\sup \varphi(\partial Q)
$$

where

$$
S_{\rho}^{+}=\left\{u \in X^{+}:\|u\|=\rho\right\}, \quad Q=\left\{s e+v: v \in X^{-}, s \geq 0,\|s e+v\| \leq r\right\} .
$$

Then for some $c \geq \kappa$, there exists a sequence $\left\{u_{n}\right\} \subset X$ satisfying

$$
\varphi\left(u_{n}\right) \rightarrow c, \quad\left\|\varphi^{\prime}\left(u_{n}\right)\right\|\left(1+\left\|u_{n}\right\|\right) \rightarrow 0 .
$$

Such a sequence is called a Cerami sequence on the level c, or a $(C)_{c}$ sequence.

Now we define a functional $\Phi$ on $E$ by

$$
\left.\Phi(u)=\frac{1}{2} \sum_{n \in \mathbb{Z}}[p(n+1) \Delta u \cdot \Delta u+L(n) u \cdot u)\right]-\sum_{n \in \mathbb{Z}} W(n, u) .
$$

For any $u \in E$, there exists an $n_{0} \in \mathbb{N}$ such that $|u(n)| \leq 1$ for $|n| \geq n_{0}$. Hence, under assumptions (PL), (W1), and (W2), the functional $\Phi$ is of class $C^{1}(E, \mathbb{R})$. Moreover,

$$
\left\langle\Phi^{\prime}(u), v\right\rangle=\sum_{n \in \mathbb{Z}}[p(n+1) \Delta u \cdot \Delta v+L(n) u \cdot v]-\sum_{n \in \mathbb{Z}} \nabla W(n, u) \cdot v, \quad \forall u, v \in E .
$$

By virtue of (2.1), (2.2), (2.3), and (2.4), one has

$$
\Phi(u)=\frac{1}{2}\left(\left\|u^{+}\right\|^{2}-\left\|u^{-}\right\|^{2}\right)-\sum_{n \in \mathbb{Z}} W(n, u), \quad \forall u \in E,
$$

and

$$
\left\langle\Phi^{\prime}(u), v\right\rangle=\left(u^{+}, v\right)-\left(u^{-}, v\right)-\sum_{n \in \mathbb{Z}} \nabla W(n, u) \cdot v, \quad \forall u, v \in E
$$


Furthermore, the critical points of $\Phi$ in $E$ are solutions of system (1.1) with $u( \pm \infty)=0$; see $[6,10]$.

Let

$$
\Psi(u)=\sum_{n \in \mathbb{Z}} W(n, u), \quad \forall u \in E .
$$

Then, by standard arguments, we can prove the following two lemmas.

Lemma 2.2 Suppose that (PL), (W1), and (W2) are satisfied. Then $\Psi$ is nonnegative, weakly sequentially lower semi-continuous, and $\Psi^{\prime}$ is weakly sequentially continuous.

Lemma 2.3 Suppose that (PL), (W1), and (W2) are satisfied. Then there is a $\rho>0$ such that $\kappa:=\inf \Phi\left(S_{\rho}^{+}\right)>0$, where $S_{\rho}^{+}=\partial B_{\rho} \cap E^{+}$.

Let $m_{0}:=\inf _{n \in \mathbb{Z},|x|=1} M(n) x \cdot x$. Then (W3) implies that $m_{0}>\bar{\Lambda}$. Since $\sigma(\mathcal{A})$ is a union of closed intervals, we can choose $e \in\left[\mathcal{E}\left(m_{1}\right)-\mathcal{E}(\bar{\Lambda})\right] E \subseteq E^{+}$, where $\bar{\Lambda}<m_{1}<m_{0}$. Thus,

$$
\bar{\Lambda}\|e\|_{2}^{2} \leq\|e\|^{2} \leq m_{1}\|e\|_{2}^{2}<m_{0}\|e\|_{2}^{2} \leq \sum_{n \in \mathbb{Z}} M(n) e \cdot e .
$$

Lemma 2.4 Suppose that (PL), (W1), (W2), and (W3) are satisfied. Then there is a $r_{0}>0$ such that $\sup \Phi(\partial Q) \leq 0$, where

$$
Q=\left\{w+s e: w \in E^{-}, s \geq 0,\|w+s e\| \leq r_{0}\right\} .
$$

Proof Obviously, $\Phi(w) \leq 0$ for $w \in E^{-}$. It is sufficient to show that $\Phi(w+t e) \leq 0$ for $t \geq 0$, $w \in E^{-}$and $\|w+t e\| \geq r$ for large $r>0$. Arguing indirectly, assume that for some sequence $\left\{w_{k}+t_{k} e\right\} \subset E^{-} \oplus \mathbb{R}^{+} e$ with $\left\|w_{k}+t_{k} e\right\| \rightarrow \infty, \Phi\left(w_{k}+t_{k} e\right) \geq 0$ for all $k \in \mathbb{N}$. Set $v_{k}=\left(w_{k}+\right.$ $\left.t_{k} e\right) /\left\|w_{k}+t_{k} e\right\|=v_{k}^{-}+s_{k} e$, then $\left\|v_{k}^{-}+s_{k} e\right\|=1$. Passing to a subsequence, we may assume that $v_{k} \rightarrow v$ in $E$, then $v_{k}(n) \rightarrow v(n)$ for all $n \in \mathbb{Z}, v_{k}^{-} \rightarrow v^{-}$in $E, s_{k} \rightarrow s$, and

$$
\frac{\Phi\left(w_{k}+t_{k} e\right)}{\left\|w_{k}+t_{k} e\right\|^{2}}=\frac{s_{k}^{2}}{2}\|e\|^{2}-\frac{1}{2}\left\|v_{k}^{-}\right\|^{2}-\sum_{n \in \mathbb{Z}} \frac{W\left(n, w_{k}+t_{k} e\right)}{\left\|w_{k}+t_{k} e\right\|^{2}} \geq 0 .
$$

Clearly, (2.15) yields $s>0$. By virtue of (2.13), there exists a finite set $\Pi \subset \mathbb{Z}$ such that

$$
s^{2}\|e\|^{2}-\left\|v^{-}\right\|^{2}-\sum_{n \in \Pi} M(n)\left(s e+v^{-}\right) \cdot\left(s e+v^{-}\right)<0 .
$$

From (W3) and (2.15), one has

$$
\begin{aligned}
0 & \leq \frac{s_{k}^{2}}{2}\|e\|^{2}-\frac{1}{2}\left\|v_{k}^{-}\right\|^{2}-\sum_{n \in \Pi} \frac{W\left(n, w_{k}+t_{k} e\right)}{\left\|w_{k}+t_{k} e\right\|^{2}} \\
& =\frac{s_{k}^{2}}{2}\|e\|^{2}-\frac{1}{2}\left\|v_{k}^{-}\right\|^{2}-\frac{1}{2} \sum_{n \in \Pi} M(n) v_{k} \cdot v_{k}-\sum_{n \in \Pi} \frac{W_{\infty}\left(n, w_{k}+t_{k} e\right)}{\left\|w_{k}+t_{k} e\right\|^{2}} .
\end{aligned}
$$

Clearly, $\left|W_{\infty}(n, x)\right| \leq c_{0}|x|^{2}$ for some $c_{0}>0$ and $W_{\infty}(n, x) /|x|^{2} \rightarrow 0$ as $|x| \rightarrow \infty$. Since $v_{k} \rightarrow$ $v$ in $E$, then $v_{k}(n) \rightarrow v(n)$ for $n \in \Pi$. Hence, one has 


$$
\begin{aligned}
\sum_{n \in \Pi} \frac{W_{\infty}\left(n, w_{k}+t_{k} e\right)}{\left\|w_{k}+t_{k} e\right\|^{2}} & =\sum_{n \in \Pi} \frac{W_{\infty}\left(n, w_{k}+t_{k} e\right)}{\left|w_{k}+t_{k} e\right|^{2}}\left|v_{k}\right| \\
& \leq c_{0} \sum_{n \in \Pi,|v(n)|=0}\left|v_{k}\right|+\sum_{n \in \Pi,|v(n)| \neq 0} \frac{W_{\infty}\left(n, w_{k}+t_{k} e\right)}{\left|w_{k}+t_{k} e\right|^{2}}\left|v_{k}\right| \\
& =o(1) .
\end{aligned}
$$

Hence

$$
0 \leq s^{2}\|e\|^{2}-\left\|v^{-}\right\|^{2}-\sum_{n \in \Pi} M(n)\left(s e+v^{-}\right) \cdot\left(s e+v^{-}\right)
$$

a contradiction to $(2.16)$.

Lemma 2.5 Suppose that (PL), (W1), (W2) and (W3) are satisfied. Then there exist a constant $c>0$ and a sequence $\left\{u_{k}\right\} \subset E$ satisfying

$$
\Phi\left(u_{k}\right) \rightarrow c, \quad\left\|\Phi^{\prime}\left(u_{k}\right)\right\|\left(1+\left\|u_{k}\right\|\right) \rightarrow 0 .
$$

Proof Lemma 2.5 is a direct corollary of Lemmas 2.1, 2.2, 2.3, and 2.4.

Lemma 2.6 Suppose that (PL), (W1), (W2), (W3), and (W4) are satisfied. Then any sequence $\left\{u_{k}\right\} \subset E$ satisfying (2.17) is bounded in $E$.

Proof In view of (2.17), there exists a constant $C_{0}>0$ such that

$$
C_{0} \geq \Phi\left(u_{k}\right)-\frac{1}{2}\left\langle\Phi^{\prime}\left(u_{k}\right), u_{k}\right\rangle=\sum_{n \in \mathbb{Z}} \widetilde{W}\left(n, u_{k}\right)
$$

To prove the boundedness of $\left\{u_{k}\right\}$, arguing by contradiction, suppose that $\left\|u_{k}\right\| \rightarrow \infty$. Let $v_{k}=u_{k} /\left\|u_{k}\right\|$. Then $\left\|v_{k}\right\|=1$. Passing to a subsequence, we may assume that $v_{k} \rightarrow v$ in $E$, then $v_{k}(n) \rightarrow v(n)$ for all $n \in \mathbb{Z}$. Let

$$
\Pi_{k}=\left\{n \in \mathbb{Z}: \frac{\left|\nabla W\left(n, u_{k}\right)\right|}{\left|u_{k}\right|} \leq \Lambda_{0}-\delta_{0}\right\} .
$$

Then by using $\Lambda_{0}\left\|v_{k}\right\|_{2}^{2} \leq\left\|v_{k}\right\|^{2}$, one has

$$
\begin{aligned}
\sum_{n \in \Pi_{k}} \frac{\left|\nabla W\left(n, u_{k}\right)\right|}{\left|u_{k}\right|}\left|v_{k}\right|\left|v_{k}^{+}-v_{k}^{-}\right| & \leq\left(\Lambda_{0}-\delta_{0}\right) \sum_{n \in \Pi_{k}}\left|v_{k}\right|\left|v_{k}^{+}-v_{k}^{-}\right| \\
& \leq\left(\Lambda_{0}-\delta_{0}\right)\left\|v_{k}\right\|_{2}^{2} \leq 1-\frac{\delta_{0}}{\Lambda_{0}}
\end{aligned}
$$

If $\delta:=\lim \sup _{k \rightarrow \infty}\left\|v_{k}\right\|_{\infty}=0$, then it follows from (W3), (W4), and (2.18) that

$$
\begin{aligned}
& \sum_{n \in \mathbb{Z} \backslash \Pi_{k}} \frac{\left|\nabla W\left(n, u_{k}\right)\right|}{\left|u_{k}\right|}\left|v_{k}\right|\left|v_{k}^{+}-v_{k}^{-}\right| \\
& \quad \leq\left\|v_{k}\right\|_{\infty}\left\|v_{k}^{+}-v_{k}^{-}\right\|_{\infty} \sum_{n \in \mathbb{Z} \backslash \Pi_{k}} \frac{\left|\nabla W\left(n, u_{k}\right)\right|}{\left|u_{k}\right|}
\end{aligned}
$$




$$
\begin{aligned}
& \leq C_{1}\left\|v_{k}\right\|_{\infty} \sum_{n \in \mathbb{Z} \backslash \Pi_{k}} \tilde{W}\left(n, u_{k}\right) \\
& \leq C_{2}\left\|v_{k}\right\|_{\infty}=o(1) .
\end{aligned}
$$

From (2.10), (2.11), (2.19), and (2.20), one gets

$$
\begin{aligned}
1+o(1) & =\frac{\left\|u_{k}\right\|^{2}-\left\langle\Phi^{\prime}\left(u_{k}\right), u_{k}^{+}-u_{k}^{-}\right\rangle}{\left\|u_{k}\right\|^{2}} \\
& \leq \sum_{n \in \mathbb{Z}} \frac{\left|\nabla W\left(n, u_{k}\right)\right|}{\left|u_{k}\right|}\left|v_{k}\right|\left|v_{k}^{+}-v_{k}^{-}\right| \\
& =\sum_{n \in \Pi_{k}} \frac{\left|\nabla W\left(n, u_{k}\right)\right|}{\left|u_{k}\right|}\left|v_{k}\right|\left|v_{k}^{+}-v_{k}^{-}\right|+\sum_{n \in \mathbb{Z} \backslash \Pi_{k}} \frac{\left|\nabla W\left(n, u_{k}\right)\right|}{\left|u_{k}\right|}\left|v_{k}\right|\left|v_{k}^{+}-v_{k}^{-}\right| \\
& \leq 1-\frac{\delta_{0}}{\Lambda_{0}}+o(1),
\end{aligned}
$$

a contradiction. Thus $\delta>0$.

Going if necessary to a subsequence, we may assume the existence of $n_{k} \in \mathbb{Z}$ such that

$$
\left|v_{k}\left(n_{k}\right)\right|=\left\|v_{k}\right\|_{\infty}>\frac{\delta}{2}
$$

Choose integers $i_{k}$ and $m_{k}$ with $0 \leq m_{k} \leq N-1$ such that $n_{k}=i_{k} N+m_{k}$. Let $\tilde{v}_{k}(n)=v_{k}(n+$ $\left.i_{k} N\right)$, then

$$
\left|\tilde{v}_{k}\left(m_{k}\right)\right|>\frac{\delta}{2}, \quad \forall k \in N
$$

Now we define $\tilde{u}_{k}(n)=u_{k}\left(n+i_{k} N\right)$. Since $p(n), L(n)$, and $W(n, x)$ are $N$-periodic in $n$, then $\tilde{u}_{k} /\left\|u_{k}\right\|=\tilde{v}_{k}$ and $\left\|\tilde{u}_{k}\right\|=\left\|u_{k}\right\|$. Passing to a subsequence, we have $\tilde{v}_{k} \rightarrow \tilde{v}$ in $E$, then $\tilde{v}_{k}(n) \rightarrow$ $\tilde{v}(n)$ for all $n \in \mathbb{Z}$. Obviously, (2.22) implies that $\tilde{v}(n) \neq 0$ for some $n \in\{0,1, \ldots, N-1\}$. Let

$$
E_{0}=\{u \in E:\{n \in \mathbb{Z}:|u(n)|>0\} \text { is finite set }\} .
$$

For any $\phi \in E_{0}$, there exists an $n_{0} \in \mathbb{N}$ such that $\phi(n)=0$ for all $|n|>n_{0}$. Setting $\phi_{k}(n)=$ $\phi\left(n-i_{k} N\right)$, then it follows from (W3) and (2.9) that

$$
\begin{aligned}
& \frac{\left\langle\Phi^{\prime}\left(u_{k}\right), \phi_{k}\right\rangle}{\left\|u_{k}\right\|} \\
& =\sum_{n \in \mathbb{Z}}\left[p(n+1) \Delta v_{k} \cdot \Delta \phi_{k}+L(n) v_{k} \cdot \phi_{k}-\frac{\nabla W\left(n, u_{k}\right) \cdot \phi_{k}}{\left\|u_{k}\right\|}\right] \\
& =\sum_{n \in \mathbb{Z}}\left[p(n+1) \Delta v_{k} \cdot \Delta \phi_{k}+(L(n)-M(n)) v_{k} \cdot \phi_{k}-\frac{\nabla W_{\infty}\left(n, u_{k}\right) \cdot \phi_{k}}{\left\|u_{k}\right\|}\right] \\
& =\sum_{n \in \mathbb{Z}}\left[p(n+1) \Delta \tilde{v}_{k} \cdot \Delta \phi+(L(n)-M(n)) \tilde{v}_{k} \cdot \phi-\frac{\nabla W_{\infty}\left(n, \tilde{u}_{k}\right) \cdot \phi}{\left\|\tilde{u}_{k}\right\|}\right] .
\end{aligned}
$$


Note that

$$
\left|\sum_{n \in \mathbb{Z}} \frac{\nabla W_{\infty}\left(n, \tilde{u}_{k}\right) \cdot \phi}{\left\|\tilde{u}_{k}\right\|}\right|=\left|\sum_{|n| \leq n_{0}} \frac{\nabla W_{\infty}\left(n, \tilde{u}_{k}\right) \cdot \phi}{\left\|\tilde{u}_{k}\right\|}\right| \leq \sum_{|n| \leq n_{0}} \frac{\left|\nabla W_{\infty}\left(n, \tilde{u}_{k}\right)\right|}{\left|\tilde{u}_{k}\right|}\left|\tilde{v}_{k}\right||\phi|=o(1) .
$$

Hence, it follows from (2.17) and (2.23) that

$$
\sum_{n \in \mathbb{Z}}\left[p(n+1) \triangle \tilde{v}_{k} \cdot \Delta \phi+(L(n)-M(n)) \tilde{v}_{k} \cdot \phi\right]=o(1)
$$

which yields

$$
\sum_{n \in \mathbb{Z}}[p(n+1) \triangle \tilde{v} \cdot \Delta \phi+(L(n)-M(n)) \tilde{v} \cdot \phi]=0 .
$$

This shows that $\tilde{v}$ is an eigenfunction of the operator $\mathcal{B}$, where

$$
(\mathcal{B} u)(n)=\Delta[p(n) \Delta u(n-1)]-(L(n)-M(n)) u(n), \quad \forall n \in \mathbb{Z} .
$$

But $\mathcal{B}$ has only continuous spectrum in $E$. This contradiction shows that $\left\{u_{n}\right\}$ is bounded.

Proof of Theorem 1.1 In view of Lemmas 2.5 and 2.6, there exists a bounded sequence $\left\{u_{k}\right\} \subset E$ satisfying (2.17). Thus there exists a constant $C_{3}>0$ such that

$$
\sqrt{\Lambda_{0}}\left\|u_{k}\right\|_{\infty} \leq \sqrt{\Lambda_{0}}\left\|u_{k}\right\|_{2} \leq\left\|u_{k}\right\| \leq C_{3}, \quad \forall k \in \mathbb{N} .
$$

Hence, by (W1) and (W2), there exists a constant $C_{4}>0$ such that

$$
|\widetilde{W}(n, x)| \leq \frac{c \Lambda_{0}}{2 C_{3}^{2}}|x|^{2}+C_{4}|x|^{3}, \quad \forall(n, x) \in \mathbb{Z} \times \mathbb{R}^{\mathcal{N}},|x| \leq \frac{C_{3}}{\sqrt{\lambda_{0}}} .
$$

If $\delta:=\lim \sup _{k \rightarrow \infty}\left\|u_{k}\right\|_{\infty}=0$, then

$$
\sum_{n \in \mathbb{Z}}\left|u_{k}(n)\right|^{3} \leq\left\|u_{k}\right\|_{\infty} \sum_{n \in \mathbb{Z}}\left|u_{k}(n)\right|^{2} \leq \frac{C_{3}^{2}}{\Lambda_{0}}\left\|u_{k}\right\|_{\infty}=o(1) .
$$

From (2.10), (2.11), (2.17), (2.26), (2.27), and (2.28), one has

$$
\begin{aligned}
c & =\Phi\left(u_{k}\right)-\frac{1}{2}\left\langle\Phi^{\prime}\left(u_{k}\right), u_{k}\right\rangle+o(1) \\
& =\sum_{n \in \mathbb{Z}} \widetilde{W}\left(n, u_{k}\right)+o(1) \\
& \leq \frac{c \Lambda_{0}}{2 C_{3}^{2}} \sum_{n \in \mathbb{Z}}\left|u_{k}(n)\right|^{2}+C_{4} \sum_{n \in \mathbb{Z}}\left|u_{k}(n)\right|^{3}+o(1) \\
& \leq \frac{c}{2}+o(1) .
\end{aligned}
$$

This contradiction shows that $\delta>0$. 
Going if necessary to a subsequence, we may assume the existence of $n_{k} \in \mathbb{Z}$ such that

$$
\left|u_{k}\left(n_{k}\right)\right|=\left\|u_{k}\right\|_{\infty}>\frac{\delta}{2} .
$$

Choose integers $i_{k}$ and $m_{k}$ with $0 \leq m_{k} \leq N-1$ such that $n_{k}=i_{k} N+m_{k}$. Let $v_{k}(n)=u_{k}(n+$ $\left.i_{k} N\right)$, then

$$
\left|v_{k}\left(m_{k}\right)\right|>\frac{\delta}{2}, \quad \forall k \in \mathbb{N} .
$$

Since $p(n), L(n)$, and $W(n, x)$ are $N$-periodic in $n$, we have $\left\|v_{k}\right\|=\left\|u_{k}\right\|$ and

$$
\Phi\left(v_{k}\right) \rightarrow c, \quad\left\|\Phi^{\prime}\left(v_{k}\right)\right\|\left(1+\left\|v_{k}\right\|\right) \rightarrow 0 .
$$

Passing to a subsequence, we have $v_{k} \rightarrow v$ in $E, v_{k}(n) \rightarrow v(n)$ for all $n \in \mathbb{Z}$. Obviously, (2.29) implies that $v \neq 0$. It is easy to show that $\Phi^{\prime}(v)=0$.

\section{Competing interests}

The author declares that they have no competing interests.

\section{Acknowledgements}

This work is partially supported by the NNSF (No: 11471278) of China and Scientific Research Fund of Hunan Provincial Education Department (13A093).

Received: 20 October 2014 Accepted: 27 January 2015 Published online: 24 February 2015

\section{References}

1. Agarwal, RP: Difference Equations and Inequalities: Theory, Methods, and Applications, 2nd edn. Dekker, New York (2000)

2. Ahlbran, CD, Peterson, AC: Discrete Hamiltonian Systems: Difference Equations, Continued Fraction and Riccati Equations. Kluwer Academic, Dordrecht (1996)

3. Chen, WX, Yang, MB, Ding, YH: Homoclinic orbits of first order discrete Hamiltonian systems with super linear terms. Sci. China Math. 54, 2583-2596 (2011)

4. Deng, $X Q$, Cheng, G: Homoclinic orbits for second order discrete Hamiltonian systems with potential changing sign. Acta Appl. Math. 103, 301-314 (2008). doi:10.1007/s10440-008-9237-z

5. Deng, XQ, Cheng, G, Shi, HP: Subharmonic solutions and homoclinic orbits of second order discrete Hamiltonian systems with potential changing sign. Comput. Math. Appl. 58, 1198-1206 (2009)

6. Lin, $X Y, T a n g, X H$ : Existence of infinitely many homoclinic orbits in discrete Hamiltonian systems. J. Math. Anal. Appl. 373, 59-72 (2011)

7. Lin, $X Y$, Tang, $X H$ : Homoclinic orbits for discrete Hamiltonian systems with subquadratic potential. Adv. Differ. Equ. 2013, 154 (2013) http://www.advancesindifferenceequations.com/content/2013/1/154

8. Tang, $\mathrm{XH}, \mathrm{Chen}, \mathrm{J}$ : Infinitely many homoclinic orbits for a class of discrete Hamiltonian systems. Adv. Differ. Equ. 2013, 242 (2013) http://www.advancesindifferenceequations.com/content/2013/1/242

9. Tang, XH, Lin, XY, Xiao, L: Homoclinic solutions for a class of second order discrete Hamiltonian systems. J. Differ. Equ. Appl. 16, 1257-1273 (2010)

10. Tang, $X H$, Lin, $X Y$ : Existence and multiplicity of homoclinic solutions for second-order discrete Hamiltonian systems with subquadratic potential. J. Differ. Equ. Appl. 17, 1617-1634 (2011)

11. Tang, $\mathrm{XH}$, Lin, $\mathrm{XY}$ : Homoclinic solutions for a class of second order discrete Hamiltonian systems. Acta Math. Sin. 28 609-622 (2012)

12. Tang, $\mathrm{XH}$, Lin, $\mathrm{XY}$ : Infinitely many homoclinic orbits for discrete Hamiltonian systems with subquadratic potential. J. Differ. Equ. Appl. 19, 796-813 (2013)

13. Wang, $X$ : New potential condition on homoclinic orbits for a class of discrete Hamiltonian systems. Adv. Differ. Equ. 2014, 73 (2014) http://www.advancesindifferenceequations.com/content/2014/1/73

14. Yu, JS, Shi, H, Guo, ZM: Homoclinic orbits for nonlinear difference equations containing both advance and retardation. J. Math. Anal. Appl. 352, 799-806 (2009)

15. Zhang, Q: Homoclinic orbits for a class of discrete periodic Hamiltonian systems. Proc. Am. Math. Soc. (2015, in press)

16. Zhou, Z, Yu, JS: On the existence of homoclinic solutions of a class of discrete nonlinear periodic systems. J. Differ. Equ. 249, 1199-1212 (2010)

17. Zhou, Z, Yu, JS: Homoclinic solutions in periodic nonlinear difference equations with superlinear nonlinearity. Acta Math. Sin. 29, 1809-1822 (2013)

18. Zhou, Z, Yu, JS, Chen, Y: Homoclinic solutions in periodic difference equations with saturable nonlinearity. Sci. China Math. 54, 83-93 (2011) 
19. Edmunds, DE, Evans, WD: Spectral Theory and Differential Operators. Clarendon, Oxford (1987)

20. Tang, XH: Non-Nehari manifold method for asymptotically linear Schrödinger equation. J. Aust. Math. Soc. 98, 104-116 (2015)

21. Li, GB, Szulkin, A: An asymptotically periodic Schrödinger equation with indefinite linear part. Commun. Contemp. Math. 4, 763-776 (2002)

Submit your manuscript to a SpringerOpen ${ }^{\circ}$ journal and benefit from:

- Convenient online submission

Rigorous peer review

- Immediate publication on acceptance

- Open access: articles freely available online

- High visibility within the field

- Retaining the copyright to your article 